\title{
Interleaved difference-frequency-generation for mid-infrared microcomb spectral densification
}

\author{
Chengying Bao, ${ }^{1, \dagger}$ Zhiquan Yuan, ${ }^{1, \dagger}$ Heming Wang, ${ }^{1}$ Lue Wu,,${ }^{1}$ Boqiang Shen, ${ }^{1}$ \\ Keeyoon Sung, ${ }^{2}$ Stephanie Leifer, ${ }^{2}$ Qiang Lin, ${ }^{3}$ and Kerry Vahala ${ }^{1, *}$ \\ ${ }^{1}$ T. J. Watson Laboratory of Applied Physics, California Institute of Technology, Pasadena, CA 91125, USA. \\ ${ }^{2}$ Jet Propulsion Laboratory, California Institute of Technology, Pasadena, CA 91109, USA. \\ ${ }^{3}$ Department of Electrical and Computer Engineering, University of Rochester, Rochester, NY 14627, USA. \\ ${ }^{\dagger}$ These authors contributed equally to this work. \\ *vahala@caltech.edu
}

\begin{abstract}
Generation of mid-infrared combs (3.3 micron band) with GigaHertz line spacing is demonstrated by interleaved difference-frequency-generation. The method, applied to a $22 \mathrm{GHz}$ repetition-rate microcomb, is useful for spectral densification of sparse microcomb spectra. $\odot 2020$ The Author(s)
\end{abstract}

Temporal soliton formation in coherently pumped microresonators provides a reliable method to generate fully coherent microcombs that can be used in a wide range of applications including spectroscopy, optical frequency synthesis, and optical clocks [1]. In application to spectroscopy, microcombs can be an invaluable tool for detection of chemical threats and field observation of green-house gases (e.g., $\mathrm{CO}_{2}$ and $\mathrm{CH}_{4}$ ). However, on account of their small form factors, microcombs usually feature wide comb line spacings $(>10 \mathrm{GHz})$. Such a wide line spacing may cause spectral undersampling when using microcombs for gas spectroscopy. Hence, it is desirable to spectrally densify the microcombs (see Fig. 1(a) right-top panel). Soliton microcombs with GigaHertz line spacing have only been possible in the near-infrared (IR) [2]. And although there has been progress on mid-IR soliton microcombs [3], generating mid-IR microcombs with GigaHertz line spacing still presents a significant challenge. Resolving this challenge can be an important step towards scalable and portable microcomb-based mid-IR gas sensor modules.

Here, we introduce interleaved difference-frequency-generation (iDFG) (i.e., DFG between two combs with different repetition rates) to spectrally densify microcomb line spacing upon conversion to the mid-IR band. DFG is a well-known technique that leverages more mature visible and near-IR light sources to generate mid-IR light

(a)

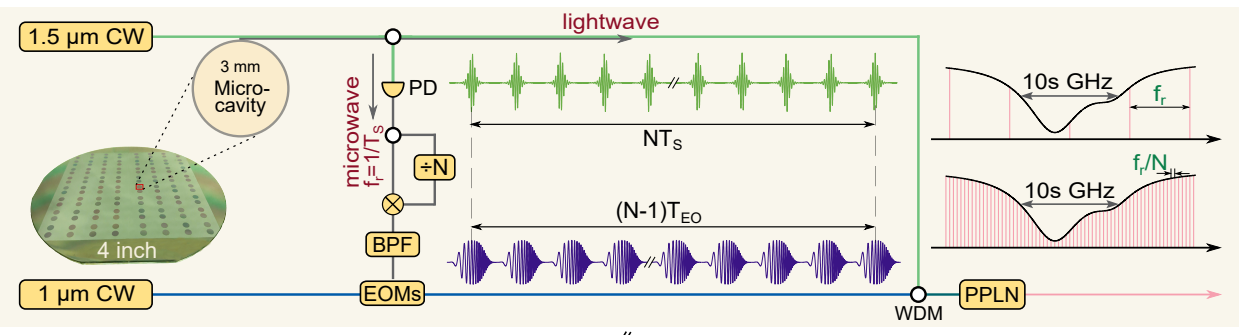

(b)

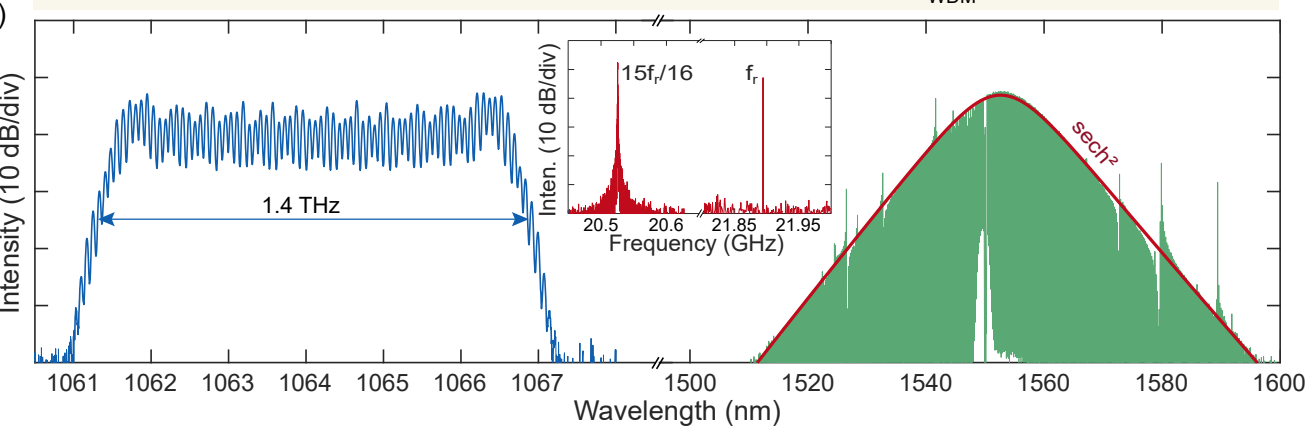

Fig. 1. (a) Experimental layout for iDFG. EOM: electro-optical modulator, BPF: bandpass filter, WDM: wavelength division multiplexer. (b) The optical spectra of the 1.55 micron soliton microcomb and the 1.06 micron EO-comb. The inset shows the repetition rate $f_{r}$ of the soliton microcomb and the derived frequency of $15 f_{r} / 16$ that drives the EO-comb. 
(a)

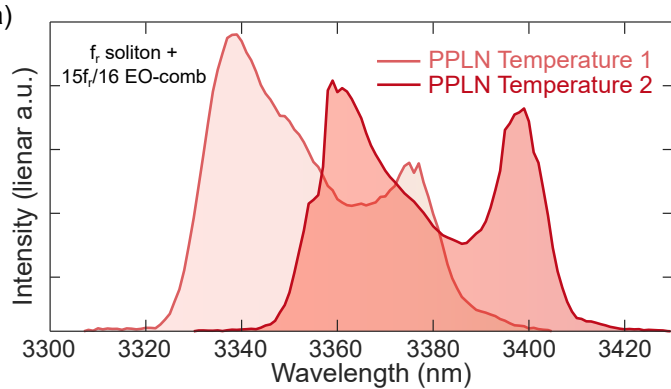

(b)

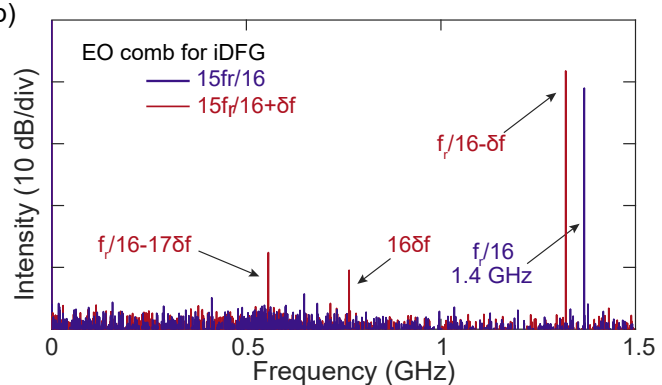

Fig. 2. (a) Spectra of the iDFG generated mid-IR comb. The comb center wavelength is shifted by changing the temperature of the PPLN crystal. Due to the limited resolution of the spectrometer, individual comb lines are not resolved. (b) Electrical spectrum of the photo detected mid-IR comb in panel (a) showing a repetition rate of $f_{r} / 16=1.4 \mathrm{GHz}$ (blue line) resulting from driving the EOcomb at $15 f_{r} / 16$. When driving the EO-comb at a frequency slightly offset from $15 f_{r} / 16$, there are additional peaks in the electrical spectrum (red lines).

usually from combs having equal repetition rates $[4,5]$. In contrast to this conventional DFG, we apply two nearIR combs with different repetition rates. If rates $f_{r}$ and $(N-1) f_{r} / N(N$ is an integer) are used then a temporal interleaving effect is created whereby the repetition rate of the generated mid-IR comb is reduced to $f_{r} / N$. A more general analysis shows that for $\Delta f_{r}=m f_{r} / n\left(\Delta f_{r}\right.$ is the repetition rate rate difference between the two near-IR combs) that $m, n$ must be mutually-prime to ensure a uniform comb line spacing in the mid-IR.

In the experiment, a 1.55 micron soliton microcomb (rate $f_{r}=22 \mathrm{GHz}$ ) is used as both a lightwave source and a microwave source. A 1.06 micron electro-optic frequency comb (EO-comb) is also used (see Fig. 1(a)). Figure 1 (b) shows the optical and electrical spectra of combs. By processing the $22 \mathrm{GHz}$ microcomb signal, a microwave frequency of $15 f_{r} / 16$ is generated and is used to drive the EO-comb (see Fig. 1(b)). The soliton microcomb is silcon-chip based and the $\mathrm{LiNbO}_{3}$ EO-comb could also be integrated [6]. When mixing the soliton microcomb and EO-comb in a PPLN crystal, a mid-IR comb at 3.3 microns is generated (Fig. 2(a)). The mid-IR comb spans about $80 \mathrm{~nm}$ which includes more than 1500 lines with a spacing of $1.4 \mathrm{GHz}$ (i.e., reduced to $f_{r} / 16$ through the iDFG process). The center wavelength of the mid-IR comb can be tuned by changing the temperature of the crystal. Due to the limited spectral resolution of the mid-IR spectrometer, a discrete comb structure is not resolvable in the spectrum. Therefore, to verify that the generated comb has a uniform line spacing, a fast mid-IR detector is used to measure the line spacing by photodetection of the comb. The electrical spectrum of the detected 3.3 micron comb shows a single peak at $f_{r} / 16=1.4 \mathrm{GHz}$ (blue line Fig. 2(b)). There are no additional RF peaks which suggests the line spacing is uniform in the mid-IR. In contrast, when choosing a non-optimal EO-comb line spacing, there are additional RF peaks observed indicating that the 3.3 micron light has a non-uniform spacing (red lines in Fig. 2(b)). In this test, the EO-comb drive frequency is derived from an independent microwave oscillator (as opposed to the microcomb) and the frequency is slightly offset $(\delta f)$ from $15 f_{r} / 16$.

In summary, we have introduced iDFG for spectral densification of mid-IR comb light generated by differencefrequency generation. 3.3 micron comb light having GigaHertz line spacing was produced from K-band rate nearIR combs. The method can be integrated using compact microcombs either alone or in combination with thin-film $\mathrm{LiNbO}_{3}$ waveguides and modulators. Such a system would be useful for field deployable and compact methane and green-house gas sensor systems.

We acknowledge support from the DTRA (HD-TRA11810047); the AFSOR (FA9550-18-1-0353) and the Resnick Institute at Caltech.

\section{References}

1. T. J. Kippenberg, A. L. Gaeta, M. Lipson, and M. L. Gorodetsky, Science 361, eaan8083 (2018)

2. M.-G. Suh and K. Vahala, Optica 5, 65-66 (2018)

3. M. Yu, Y. Okawachi, A. G. Griffith, M. Lipson, and A. L.Gaeta, Optica 3, 854-860 (2016)

4. H. Timmers, A. Kowligy, A. Lind, F. C. Cruz, N. Nader, M. Silfies, G. Ycas, T. K. Allison, P. G. Schunemann, S. B.Papp, and S. A. Diddams, Optica 5, 727-732 (2018)

5. G. Ycas, F. R. Giorgetta, E. Baumann, I. Coddington, D. Her-man, S. A. Diddams, and N. R. Newbury, Nat. Photonics 12, 202 (2018)

6. C. Wang, M. Zhang, X. Chen, M. Bertrand, A. Shams-Ansari, S. Chandrasekhar, P. Winzer, and M. Lončar, Nature 562, 101 (2018) 